\title{
Identification of candidate responders for anti-PD-L1/PD-1 immunotherapy, Rova-T therapy, or EZH2 inhibitory therapy in small-cell lung cancer
}

\author{
MOTONOBU SAITO ${ }^{1,2}$, KATSUHARU SAITO $^{2}$, KOUYA SHIRAISHI ${ }^{1}$, DAICHI MAEDA ${ }^{3}$, HIROYUKI SUZUKI ${ }^{4}$, \\ YOSHIHIRO MINAMIYA ${ }^{5}$, KOJI KONO ${ }^{2}$, TAKASHI KOHNO ${ }^{1}$ and AKITERU GOTO ${ }^{3}$ \\ ${ }^{1}$ Division of Genome Biology, National Cancer Center Research Institute, Tokyo 104-0045; ${ }^{2}$ Department of \\ Gastrointestinal Tract Surgery, Fukushima Medical University School of Medicine, Fukushima, Fukushima 960-1295; \\ ${ }^{3}$ Department of Cellular and Organ Pathology, Graduate School of Medicine, Akita University, Akita, Akita 010-8543; \\ ${ }^{4}$ Department of Chest Surgery, Fukushima Medical University School of Medicine, Fukushima, Fukushima 960-1295; \\ ${ }^{5}$ Department of Thoracic Surgery, Graduate School of Medicine, Akita University, Akita, Akita 010-8543, Japan
}

Received September 6, 2017; Accepted November 1, 2017

DOI: $10.3892 /$ mco.2017.1536

\begin{abstract}
A useful candidate for small-cell lung cancer (SCLC) therapy is immune checkpoint blockade therapy targeting programmed death-1 (PD-1) and its ligand, PD-L1. Furthermore, rovalpituzumab tesirine (Rova-T), a delta-like protein 3 (DLL3)-targeted antibody-drug conjugate, and enhancer of zeste homologue 2 (EZH2) inhibitor are expected to be the first targeted therapy for SCLC. The aim of the present study was to evaluate PD-L1, DLL3 and EZH2 expression in SCLCs to find a candidate responder to those therapies. Immunohistochemical (IHC) staining for PD-L1, DLL3 and EZH2 was performed in 20 patients with SCLC and the clinicopathological characteristics and IHC staining intensity were compared. It was demonstrated that $1 / 20$ patients (5.0\%) exhibited positive PD-L1 expression in the metastatic lesions, as well as in the primary lung tumor. DLL3 was highly expressed in $14 / 20$ patients $(70 \%)$ and EZH2 was positive in $17 / 20$ patients $(85 \%)$. None of these cases exhibited any correlation with age, sex, smoking, stage or treatment, whereas IHC staining was able to identify candidate responders to anti-PD-L1/PD-1 immunotherapy, Rova-T therapy, or EZH2 inhibitor therapy.
\end{abstract}

\section{Introduction}

Small-cell lung cancer (SCLC) is an aggressive type of lung cancer exhibiting rapid growth and widespread metastases,

Correspondence to: Dr Motonobu Saito, Division of Genome Biology, National Cancer Center Research Institute, 1-1, Tsukiji 5-chome, Chuo-ku, Tokyo 104-0045, Japan

E-mail: mosaitoh@ncc.go.jp

Key words: small-cell lung cancer, precision medicine, PD-L1, programmed death-ligand 1, DLL3, delta-like protein 3, EZH2, enhancer of zeste homologue 2 with a poor prognosis (1). Unlike therapy for lung adenocarcinoma (LADC), the treatment for SCLC has not significantly advanced over the last three decades (2-4). To understand the genomic landscape and identify candidate therapeutic targets in SCLC, large-scale genomic analyses were performed, which revealed that mutations in TP53 and $R B 1$, and amplifications of the MYC family members SOX2 and SRSF1, have been recurrently identified (5-8). Although it was reported that somatic genomic rearrangements of TP73 contribute to SCLC tumorigenesis (5), druggable gene aberrations are rarely identified. As there is no standard targeted therapy for SCLC, platinum-based doublet chemotherapy is recommended as first-line treatment for advanced SCLC; however, its effectiveness is limited (2). Therefore, there is a need for development of further treatment options for patients with SCLC.

The blockade of immune checkpoints in cancer immunotherapy has exhibited durable positive efficacy in non-small-cell lung cancer (NSCLC), particularly LADC (9-11). Recent clinical trials have further investigated the efficacy of monotherapy with nivolumab (12) and of combination therapy with platinum-based doublet regimens (13). Due to these positive treatment effects on NSCLC, the blockade of immune checkpoints is also expected to be a useful therapy for SCLC. Although efforts have been made to develop a biomarker to identify patients who may benefit from immunotherapy (14), such a biomarker has yet to be determined. Previous reports demonstrated that the programmed death-ligand 1 (PD-L1) protein in tumor cells is a potential predictive biomarker of response to anti-PD-1/PD-L1 immunotherapy $(10,15,16)$. Furthermore, PD-L1 expression in tumors was reported to be associated with improved efficacy of pembrolizumab (15) and with significantly longer progression-free and overall survival (17). We recently revealed the mechanism through which high expression of PD-L1 is caused by focal amplification of CD274, encoding the PD-L1 protein in SCLC (18). Although only a subset of SCLC tumors highly express PD-L1, such SCLC tumors may be particularly susceptible to immune checkpoint blockade therapy. Therefore, the aim of the present 
study was to investigate PD-L1 expression in an independent cohort of SCLCs, in order to identify a candidate responder to PD-L1 blockade therapy.

Delta-like protein 3 (DLL3) and enhancer of zeste homologue 2 (EZH2) expression was also investigated in SCLC tumors. DLL3 is highly expressed in the majority of SCLCs and inhibits the Notch receptor pathway, promoting SCLC tumorigenesis (19). A recent clinical trial demonstrated that rovalpituzumab tesirine (Rova-T), a DLL3-targeted antibody-drug conjugate, exhibited marked antitumor activity and durability in recurrent or refractory SCLC (20). EZH2 is also highly expressed in SCLC, and its inhibition by EZH2 inhibitor enhances the effectiveness of current standard chemotherapy (21). Higher expression of DLL3 and EZH2 in SCLC was associated with a higher rate of response to Rova-T and EZH2 inhibitor, respectively; thus, DLL3 and EZH2 expression may be a candidate predictive biomarker for SCLC treatment.

\section{Patients and methods}

Patients. The present study included 20 primary and two metastatic tumors obtained from 20 SCLC patients at surgery or autopsy performed between 1991 and 2013 at Akita University (Akita, Japan). We retrospectively collected information regarding age, sex, ethnicity, pathological TNM stage $(22,23)$, and smoking status. The present study was approved by the Ethics Committee of Akita University (reference nos. 1241 and 1246) and written informed consent was obtained from all patients.

Immunohistochemical (IHC) staining and evaluation. IHC staining for PD-L1 was performed as previously described (18). In addition, IHC staining for DLL3 and EZH2 were performed. Briefly, IHC staining was performed on 4- $\mu \mathrm{m}$ paraffin-embedded histological sections that had been fixed in $10 \%$ buffered formalin using a polymer peroxidase method (EnVision+/HRP; Dako; Agilent Technologies, Inc., Santa Clara, CA, USA). Following deparaffinization with xylene and rehydration using a descending alcohol series, the tissue sections were treated with $0.3 \%$ hydrogen peroxide in methanol for $30 \mathrm{~min}$ at room temperature to block endogenous peroxidase activity. Following rinsing in PBS, the sections were incubated with rabbit monoclonal anti-PD-L1 (1:400; cat. no. 13684, E1L3N; Cell Signaling Technology, Danvers, MA, USA), rabbit polyclonal anti-DLL3 (1:100; ab103102, Abcam, Cambridge, MA, USA), and rabbit monoclonal anti-EZH2 (1:100; cat. no. 12408, D2C9, Cell Signaling Technology) at $4^{\circ} \mathrm{C}$ overnight. An additional wash in PBS was followed by treatment with a ready-to-use peroxidase-labeled polymer conjugated to goat anti-rabbit immunoglobulins (catalog no. SM801; EnVision+ kit; Dako; Agilent Technologies, Inc.) as the secondary antibody for $30 \mathrm{~min}$ at room temperature. The staining was visualized with diaminobenzidine, followed by counterstaining with hematoxylin.

IHC staining for PD-L1, DLL3 and EZH2 was evaluated by two independent observers (M.S. and A.G.), including an expert pathologist (A.G.). For the evaluation of PD-L1 and EZH2, the H-score method was used (18). Briefly, staining percentages $(0-100 \%)$ and the intensity $(0$, negative; 1 , very weak; 2, moderate; and 3, strong expression) in tumor cells were evaluated, and immunostained slides were scored ranging
Table I. Patient characteristics of this study cohort $(n=20)$.

\begin{tabular}{lc}
\hline Characteristics & No. $(\%)$ \\
\hline Age, years & \\
Mean (range) & $68.0(45-82)$ \\
Sex & \\
Male & $20(100)$ \\
Female & $0(0)$ \\
Ethnicity (\%) & \\
Asian & $20(100)$ \\
Caucasian & $0(0)$ \\
African american & $0(0)$ \\
Smoking status & \\
Never smoker & $1(5)$ \\
Current or former smoker (pack years) & $19(95)$ \\
$<20$ & $17(85)$ \\
$\geq 20$ & $0(0)$ \\
Unknown & $2(10)$ \\
TNM stage & \\
I & $6(30)$ \\
II & $1(5)$ \\
III & $8(40)$ \\
IV & $5(25)$ \\
\hline
\end{tabular}

from 0 to 300 by multiplying the percentage of tumor area. For the evaluation of PD-L1, the score was divided into two intensity levels (positive, $\geq 3$; and negative, $0-2$ ) due to antibody specificity (18). For the evaluation of EZH2, the score was divided into two intensity levels (positive, $>100$; and negative, $\leq 100$ ), according to the previous study (24).

For the evaluation of DLL3, cytoplasmic or membranous staining at any intensity in the tumor cells was scored. The staining percentage (0-100\%) was then evaluated and divided into two intensity levels (high, $\geq 50 \%$; and low, $0-49 \%$ ), according to the previous study (20).

\section{Results}

PD-L1 expression in SCLC. We previously reported that a subset $(4 / 210,1.9 \%)$ of SCLC cases exhibited high expression of PD-L1 caused by high-level amplification of CD274 (18). As PD-L1-positive cases are rarely identified in SCLC, we further expanded the investigation to include 20 Japanese patients with SCLC (Table I). We performed IHC analyses of PD-L1 protein expression with the same anti-PD-L1 antibody used in our previous study (18), and identified one case $(1 / 20,5.0 \%)$ with positive PD-L1 expression (Fig. 1). This patient (AK014) had liver and lymph node metastases, and the metastatic tumors were positive for PD-L1 expression.

DLL3 and EZH2 expression in SCLC. IHC staining was next performed for DLL3 andEZH2 to identify candidate responders for Rova-T and EZH2 inhibitor therapy (Figs. 1 and 2). DLL3 expression of $\geq 1 \%$ was observed in $18 / 20$ cases $(90 \%)$ and of $\geq 50 \%$ in $14 / 20$ cases $(70 \%)$. The staining intensity of DLL3 was 


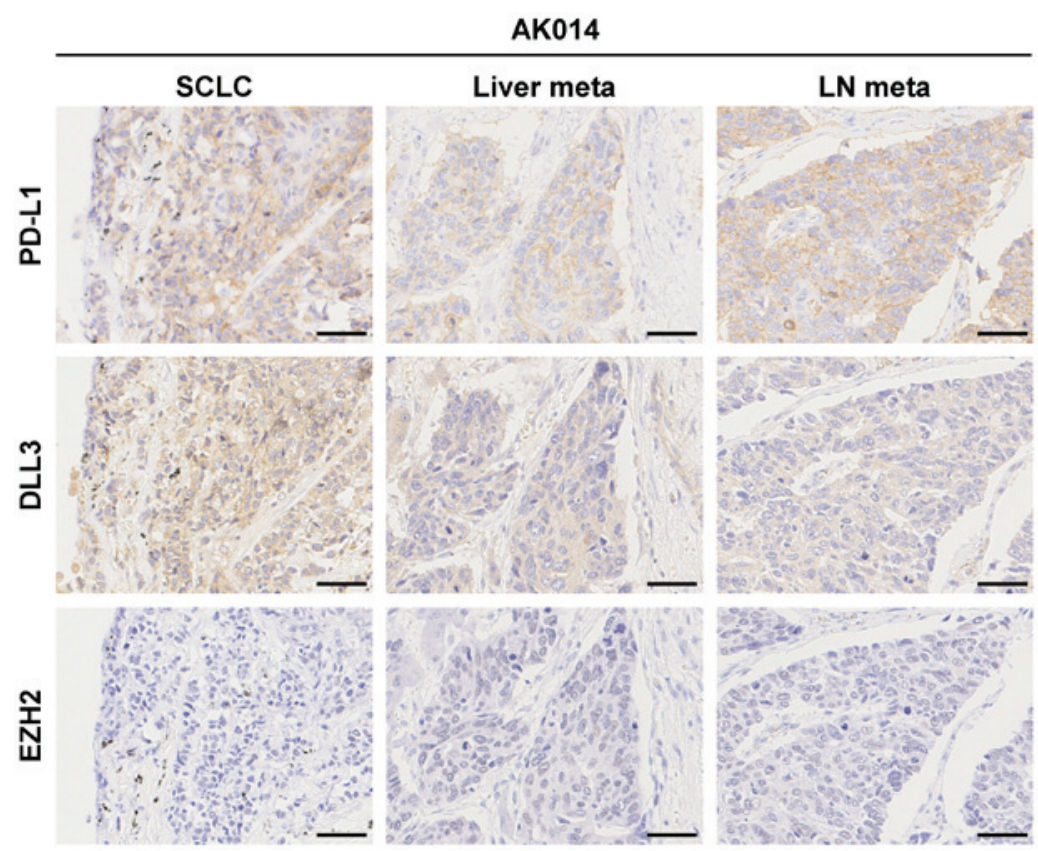

Figure 1. Immunohistochemical staining of PD-L1, DLL3, and EZH2 in SCLC. Positive expression for PD-L1, high expression for DLL3, and negative expression for EZH2 in SCLC and metastatic liver tumor and lymph node in a patient (AK014). Scale bars, $50 \mu \mathrm{m}$. SCLC, small-cell lung cancer; PD-L1, programmed death-ligand 1; DLL3, delta-like protein 3; EZH2, enhancer of zeste homologue 2; meta, metastasis.
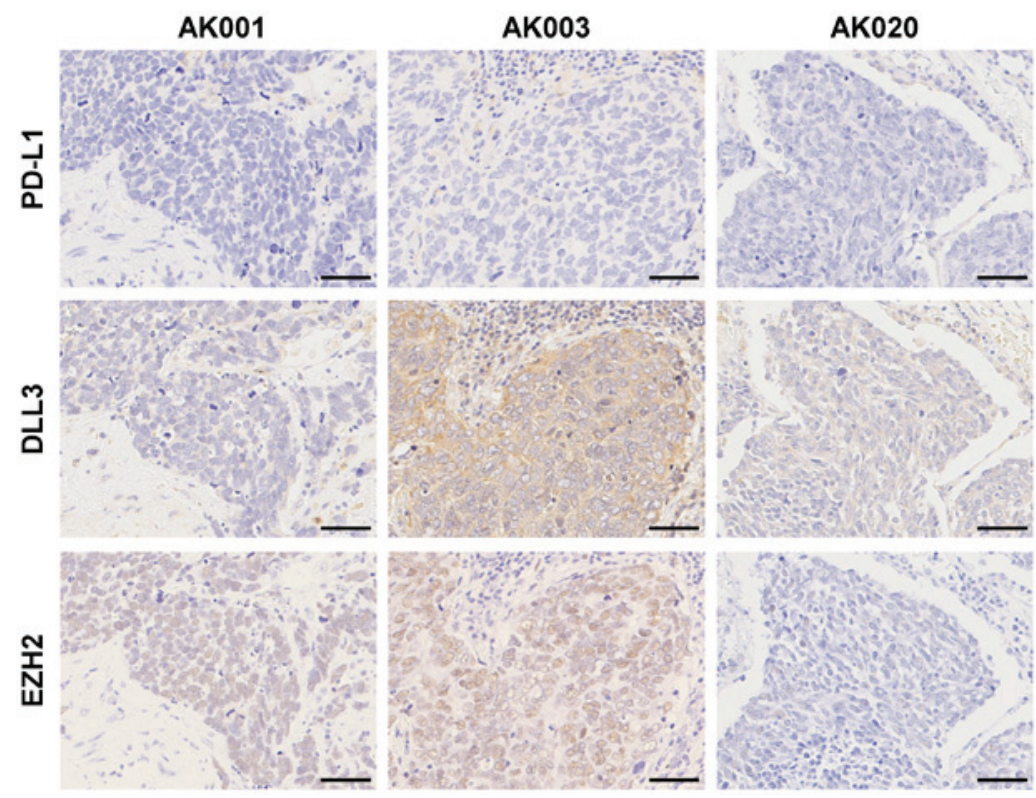

Figure 2. Immunohistochemical staining of PD-L1, DLL3, and EZH2 in SCLC. Negative expression for PD-L1, low expression of DLL3, and positive expression for EZH2 in a patient (AK001). Negative expression for PD-L1, high expression of DLL3, and positive expression for EZH2 in a patient (AK003). Negative expression for PD-L1, low expression of DLL3, and negative expression for EZH2 in a patient (AK020). Scale bars, $50 \mu \mathrm{m}$. SCLC, small-cell lung cancer; PD-L1, programmed death-ligand 1; DLL3, delta-like protein 3; EZH2, enhancer of zeste homologue 2.

consistent with a previous report from the USA (Table II) (20). EZH2 was positively expressed in $17 / 20$ patients $(85 \%)$ in our cohort. A case with positive PD-L1 expression exhibited high DLL3 and negative EZH2 expression in a metastatic liver tumor and a lymph node metastasis, as well as in a primary SCLC tumor (Fig. 1).

Therapeutic possibilities for $S C L C$. The clinicopathological characteristics, treatment history and results of IHC staining for PD-L1, DLL3 and EZH2 in the 20 cases are summarized in Table III. The cases with high DLL3 or positive EZH2 expression did not have any specific characteristics associated with age, sex, smoking, stage or treatment (Table III). Among the 20 cases, 1 exhibited positive PD-L1 expression, 14 exhibited high DLL3 expression, and 17 exhibited positive EZH2 expression. This result suggested that PD-L1 blockade therapy, Rova-T therapy, or EZH2 inhibitor therapy, may be used in $19 / 20$ cases (95\%). 
Table II. Comparison of delta-like protein 3 expression in small-cell lung cancer.

\begin{tabular}{lcc}
\hline Positively stained tumor cells $(\%)$ & Present study, $\mathrm{n} /$ total $(\%)(\mathrm{n}=20)$ & Rudin et al (20), $\mathrm{n} /$ total $(\%)(\mathrm{n}=48)$ \\
\hline$\geq 1$ & $18 / 20(90)$ & $42 / 48(88)$ \\
$\geq 50$ & $14 / 20(70)$ & $32 / 48(67)$ \\
\hline
\end{tabular}

Table III. Clinicopathological characteristics and IHC staining results of SCLC.

\begin{tabular}{|c|c|c|c|c|c|c|c|c|c|}
\hline \multirow{2}{*}{$\begin{array}{l}\text { Patient } \\
\text { ID }\end{array}$} & \multirow{2}{*}{$\begin{array}{l}\text { Age, } \\
\text { years }\end{array}$} & \multirow[b]{2}{*}{ Sex } & \multirow{2}{*}{$\begin{array}{c}\text { Smoking } \\
\text { (pack years) }\end{array}$} & \multirow[b]{2}{*}{ pStage } & \multirow{2}{*}{$\begin{array}{c}1 \mathrm{st} \\
\text { treatment }\end{array}$} & \multirow{2}{*}{$\begin{array}{l}\text { 2nd } \\
\text { treatment }\end{array}$} & \multicolumn{3}{|c|}{ IHC staining } \\
\hline & & & & & & & PD-L1 & DLL3 & EZH2 \\
\hline AK001 & 66 & Male & 46 & I & Surgery & & Negative & Low & Positive \\
\hline AK002 & 80 & Male & 65 & III & Surgery & & Negative & High & Positive \\
\hline AK003 & 82 & Male & 58 & I & Surgery & & Negative & High & Positive \\
\hline AK004 & 79 & Male & 40 & I & Surgery & & Negative & High & Positive \\
\hline AK005 & 79 & Male & 87 & $\mathrm{I}$ & Surgery & & Negative & Low & Positive \\
\hline AK006 & 45 & Male & 25 & II & Chemotherapy & Surgery & Negative & High & Positive \\
\hline AK007 & 77 & Male & N/A & III & Surgery & & Negative & Low & Positive \\
\hline AK008 & 60 & Male & 40 & $\mathrm{I}$ & Surgery & & Negative & High & Positive \\
\hline AK009 & 77 & Male & 53 & I & Surgery & & Negative & High & Positive \\
\hline AK010 & 71 & Male & 25 & III & Surgery & & Negative & Low & Positive \\
\hline AK011 & 74 & Male & 120 & IV & Chemotherapy/radiation & & Negative & High & Positive \\
\hline AK012 & 59 & Male & 50 & IV & Chemotherapy & & Negative & High & Positive \\
\hline AK013 & 63 & Male & 75 & III & Chemotherapy & & Negative & High & Positive \\
\hline AK014 & 68 & Male & 0 & IV & Radiation & & Positive & High & Negative \\
\hline AK015 & 74 & Male & 100 & III & Chemotherapy & & Negative & High & Positive \\
\hline AK016 & 68 & Male & 23 & IV & Chemotherapy/radiation & & Negative & High & Positive \\
\hline AK017 & 64 & Male & 50 & III & Chemotherapy/radiation & & Negative & High & Positive \\
\hline AK018 & 47 & Male & 60 & III & Chemotherapy/radiation & & Negative & Low & Positive \\
\hline AK019 & 59 & Male & 40 & IV & Chemotherapy/radiation & & Negative & High & Negative \\
\hline AK020 & 68 & Male & N/A & III & Chemotherapy & & Negative & Low & Negative \\
\hline
\end{tabular}

IHC, immunohistochemical; SCLC, small-cell lung cancer; PD-L1, programmed death-ligand 1; DLL3, delta-like protein 3; EZH2, enhancer of zeste homologue 2 .

\section{Discussion}

In the present study, it was reconfirmed that tumor expression of PD-L1 is rarely found to be upregulated at the protein level in SCLC. IHC analyses of PD-L1 protein expression was performed, employing the PD-L1 antibody E1L3 N, which was used in our previous study (18). The specificity of this antibody for PD-L1 was considered as high, since one case with high-level focal CD274 amplification and high transcript levels only exhibited positive PD-L1 protein expression in our previous study (18). Therefore, we concluded that focal CD274 amplification is associated with high PD-L1 antigen expression (18). The present study evaluated PD-L1 expression in cases with SCLC; however, the amplification of CD274 was not investigated due to the lack of frozen tissue samples.

Anti-PD-1/PD-L1 immunotherapy, alone or in combination with other treatment modalities, exhibits a significant efficacy for patients with various malignant tumors, including NSCLC.
Predictive biomarkers, enabling the selection of patients who will benefit the most from PD-1/PD-L1-targeted therapy and the prevention of adverse events, are required to further increase positive outcomes. Although several studies have attempted to develop a predictive biomarker using IHC, including staining for PD-L1, there is currently no reliable predictive biomarker of this immunotherapy due to cancer immune complexity. In addition, PD-L1 antibodies used in IHC staining differ among various studies and different cut-off values of PD-L1 positivity are used, making it difficult to compare results across studies. Indeed, different companion antibodies of PD-L1 made by various pharmaceutical manufacturers have been used in clinical trials (25). Although PD-L1 expression was evaluated by IHC staining of SCLC cells in the present study, it has not been established whether PD-L1 expression is correlated with clinical response and outcome.

DLL3 expression was also evaluated in SCLC tumors. DLL3 is a novel druggable target of Rova-T. Rova-T is a 
first-in-class antibody-drug conjugate directed against DLL3, and early-phase clinical trials of Rova-T assessed DLL3 expression by IHC staining. While objective responses were recorded in patients with high DLL3 expression, those with low DLL3 expression had no recorded objective responses (20). Therefore, DLL3 expression may be a therapeutic biomarker, as well as a therapeutic target. In fact, it was reported that DLL3 was expressed on the surface of tumor cells in $~ 85 \%$ of SCLC patients $(19,20)$. Consistently, DLL3 was highly expressed in our Japanese SCLC patients, and may be expected to be a candidate responder to Rova-T therapy. In addition to DLL3, EZH2 expression was evaluated in SCLC tumors. EZH2 is also expected to be a candidate therapeutic target for SCLC. However, no clinical trials targeting EZH2 in SCLC patients are currently underway.

The early success of Rova-T is accentuating the significance of targeted therapy for improving the prognosis of patients with SCLC. Thus, EZH2 inhibition, as well as Rova-T therapy, may be an option for patients with recurrent SCLC. In cases where first-line treatment results in failure, monitoring the status of PD-L1, DLL3, or EZH2 in recurrent or metastatic tumors may serve as the next regimen.

In conclusion, PD-L1, DLL3 and EZH2 expression was evaluated in additional SCLC patients following our previous study, to investigate the adoption of precision medicine. In addition to anti-PD-1/PD-L1 immunotherapy, Rova-T therapy or other DLL3- and EZH2-targeted drugs may be expected to be proven useful for the treatment of SCLC patients.

\section{Acknowledgements}

The present study was supported by JSPS KAKENHI (grant no. $15 \mathrm{~K} 10275)$.

\section{References}

1. Govindan R, Page N, Morgensztern D, Read W, Tierney R, Vlahiotis A, Spitznagel EL and Piccirillo J: Changing epidemiology of small-cell lung cancer in the United States over the last 30 years: Analysis of the surveillance, epidemiologic and end results database. J Clin Oncol 24: 4539-4544, 2006.

2. Sabari JK, Lok BH, Laird JH, Poirier JT and Rudin CM: Unravelling the biology of SCLC: Implications for therapy. Nat Rev Clin Oncol 14: 549-561, 2017.

3. Koinis F, Kotsakis A and Georgoulias V: Small cell lung cancer (SCLC): No treatment advances in recent years. Transl Lung Cancer Res 5: 39-50, 2016.

4. Saito M, Suzuki H, Kono K, Takenoshita S and Kohno T: Treatment of lung adenocarcinoma by molecular-targeted therapy and immunotherapy. Surg Today, 2017.

5. George J, Lim JS, Jang SJ, Cun Y, Ozretić L, Kong G, Leenders F, Lu X, Fernández-Cuesta L, Bosco G, et al: Comprehensive genomic profiles of small cell lung cancer. Nature 524: 47-53, 2015.

6. Iwakawa R, Kohno T, Totoki Y, Shibata T, Tsuchihara K, Mimaki S, Tsuta K, Narita Y, Nishikawa R, Noguchi M, et al: Expression and clinical significance of genes frequently mutated in small cell lung cancers defined by whole exome/RNA sequencing. Carcinogenesis 36: 616-621, 2015.

7. IwakawaR, TakenakaM,Kohno T, Shimada Y, Totoki Y, ShibataT, Tsuta K, Nishikawa R, Noguchi M, Sato-Otsubo A, et al: Genome-wide identification of genes with amplification and/or fusion in small cell lung cancer. Genes Chromosomes Cancer 52 : 802-816, 2013
8. Semenova EA, Nagel R and Berns A: Origins, genetic landscape and emerging therapies of small cell lung cancer. Genes Dev 29: 1447-1462, 2015.

9. Brahmer JR, Tykodi SS, Chow LQ, Hwu WJ, Topalian SL, Hwu P, Drake CG, Camacho LH, Kauh J, Odunsi K, et al: Safety and activity of anti-PD-L1 antibody in patients with advanced cancer. N Engl J Med 366: 2455-2465, 2012.

10. Topalian SL, Hodi FS, Brahmer JR, Gettinger SN, Smith DC, McDermott DF, Powderly JD, Carvajal RD, Sosman JA, Atkins MB, et al: Safety, activity and immune correlates of anti-PD-1 antibody in cancer. N Engl J Med 366: 2443-2454, 2012.

11. Borghaei H, Paz-A res L, Horn L, Spigel DR, Steins M, Ready NE, Chow LQ, Vokes EE, Felip E, Holgado E, et al: Nivolumab versus docetaxel in advanced nonsquamous non-small-cell lung cancer. N Engl J Med 373: 1627-1639, 2015.

12. Gettinger S, Rizvi NA, Chow LQ, Borghaei H, Brahmer J, Ready N, Gerber DE, Shepherd FA, Antonia S, Goldman JW, et al: Nivolumab monotherapy for first-line treatment of advanced non-small-cell lung cancer. J Clin Oncol 34: 2980-2987, 2016.

13. Rizvi NA, Hellmann MD, Brahmer JR, Juergens RA, Borghaei H, Gettinger S, Chow LQ, Gerber DE, Laurie SA, Goldman JW, et al: Nivolumab in combination with platinum-based doublet chemotherapy for first-line treatment of advanced non-small-cell lung cancer. J Clin Oncol 34: 2969-2979, 2016.

14. Herbst RS, Soria JC, Kowanetz M, Fine GD, Hamid O, Gordon MS, Sosman JA, McDermott DF, Powderly JD, Gettinger SN, et al: Predictive correlates of response to the anti-PD-L1 antibody MPDL3280A in cancer patients. Nature 515: 563-567, 2014.

15. Garon EB, Rizvi NA, Hui R, Leighl N, Balmanoukian AS, Eder JP, Patnaik A, Aggarwal C, Gubens M, Horn L, et al: KEYNOTE-001 Investigators: Pembrolizumab for the treatment of non-small-cell lung cancer. N Engl J Med 372: 2018-2028, 2015.

16. Taube JM, Klein A, Brahmer JR, Xu H, Pan X, Kim JH, Chen L, Pardoll DM, Topalian SL and Anders RA: Association of PD-1, PD-1 ligands and other features of the tumor immune microenvironment with response to anti-PD-1 therapy. Clin Cancer Res 20: 5064-5074, 2014.

17. Reck M, Rodríguez-Abreu D, Robinson AG, Hui R, Csőszi T, Fülöp A, Gottfried M, Peled N, Tafreshi A, Cuffe S, et al: KEYNOTE-024 Investigators: Pembrolizumab versus chemotherapy for PD-L1-positive non-small-cell lung cancer. N Engl J Med 375: 1823-1833, 2016.

18. George J, Saito M, Tsuta K, Iwakawa R, Shiraishi K, Scheel AH, Uchida S, Watanabe SI, Nishikawa R, Noguchi M, et al: Genomic amplification of CD274 (PD-L1) in small-cell lung cancer. Clin Cancer Res 23: 1220-1226, 2017.

19. SaundersLR,BankovichAJ,Anderson WC, Aujay MA,BheddahS, Black K, Desai R, Escarpe PA, Hampl J, Laysang A, et al: A DLL3-targeted antibody-drug conjugate eradicates high-grade pulmonary neuroendocrine tumor-initiating cells in vivo. Sci Transl Med 7: 302ra136, 2015.

20. Rudin CM, Pietanza MC, Bauer TM, Ready N, Morgensztern D, Glisson BS, Byers LA, Johnson ML, Burris HA III, Robert F, et al: Rovalpituzumab tesirine, a DLL3-targeted antibody-drug conjugate, in recurrent small-cell lung cancer: A first-in-human, first-in-class, open-label, phase 1 study. Lancet Oncol 18: 42-51, 2017.

21. Gardner EE, Lok BH, Schneeberger VE, Desmeules P, Miles LA, Arnold PK, Ni A, Khodos I, de Stanchina E, Nguyen T, et al: Chemosensitive relapse in small cell lung cancer proceeds through an EZH2-SLFN11 axis. Cancer Cell 31: 286-299, 2017.

22. Sobin LH and Compton CC: TNM seventh edition: What's new, what's changed: Communication from the international union against cancer and the American joint committee on cancer. Cancer 116: 5336-5339, 2010

23. Sobin LH, Gospodarowicz M and Wittekind C: Wittekind Ch: International Union Against Cancer (UICC) TNM Classification of Malignant Tumors. 7th edition. Wiley-Blackwell Oxford UK, 2009.

24. Lu C, Han HD, Mangala LS, Ali-Fehmi R, Newton CS, Ozbun L, Armaiz-Pena GN, Hu W, Stone RL, Munkarah A, et al: Regulation of tumor angiogenesis by EZH2. Cancer Cell 18: 185-197, 2010.

25. Guan J, Lim KS, Mekhail T and Chang CC: Programmed death ligand-1 (PD-L1) expression in the programmed death receptor-1 (PD-1)/PD-L1 blockade: A key player against various cancers. Arch Pathol Lab Med 141: 851-861, 2017. 